skiagram. $X$-ray hands revealed multiple lytic lesions and surrounding sclerosis (honeycombing) in metacarpals and multiple, oval, cystic lesions with variable sclerosis in radius and humerus (Fig. 2). FNAC of lymph nodes and

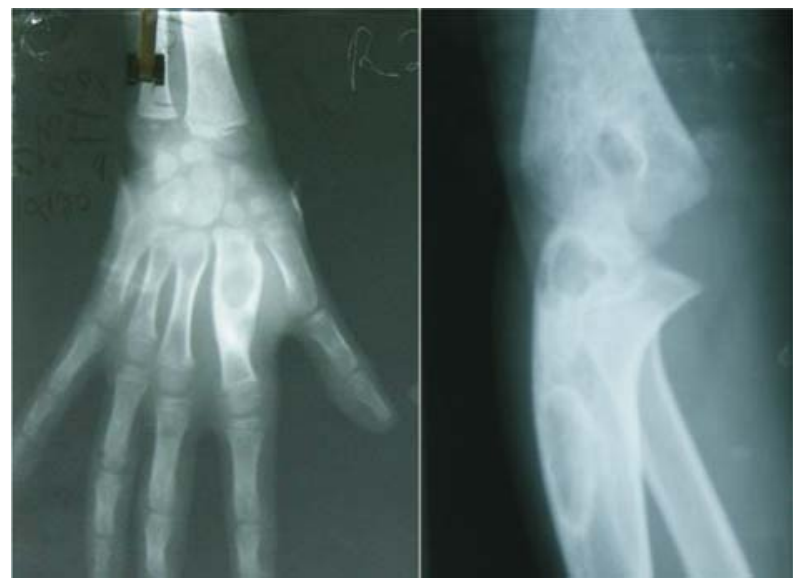

Fig. 2 Lytic lesions in bones. skin biopsy from the dorsum of hands revealed granulomatous pathology suggestive of tuberculosis. Patient responded to antitubercular treatment.

Multiple cystic tuberculosis is a rare form of tubercular osteomyelitis. The cysts may be solitary or multiple, radiolucent, round to oval, situated in the peripheral skeleton near the metaphysis. The commonly affected sites in order of frequency are spine, tibia, ulna, radius, femur, fibula and humerus. The differential diagnosis includes bacterial or fungal infection, aneurysmal bone cysts, cartilaginous tumors and osteoid osteomas. Biopsy may be required to confirm such cases. ATT is the mainstay of treatment. Surgery may be required in a few cases with extensive disease.

Rekha Harish, Ashu Jamwal and Sunil Dutt Sharma Department of Pediatrics, Govt Medical College, SMGS Hospital, Jammu, J\&K. kkrhdang@gmail.com

\section{Multiple Annular Erythematous Lesions with Trailing Scale}

A 6-year-old boy presented with recurrent pruritic scaly lesions all over trunk for last 2 years. These lesions appeared spontaneously and resolved in 4-8 weeks without any sequale. Rest of the history was non-contributory and no other family member was having similar lesions. On examination, multiple, annular erythematous lesions with trailing scale at the inner border were present on trunk (Fig. 1). Few annular lesions were found on tongue too. On enquiry, tongue lesions used to appear and disappear simultaneously with trunk lesions. Palm, sole, nail, scalp and other mucosa were unremarkable. Routine investigations and $\mathrm{KOH}$ mount did not reveal any abnormality. Clinically, the child was diagnosed as Erythema annulare centrifugum (EAC). Histopathological findings were consistent with the diagnosis. Involvement of tongue is very rare. The incidence of disease peaks in the fifth decade.

Erythema annulare centrifugum (EAC) is the most common figurate erythema and is characterized by solitary or multiple, annular or polycyclic lesions that grow slowly (2-3 mm/day); with a trailing scale at the inner border of the annular erythema. The majority of cases are idiopathic; some association with dermatophytosis, infections, drugs, foods (tomato) and malignancy have been noted. The

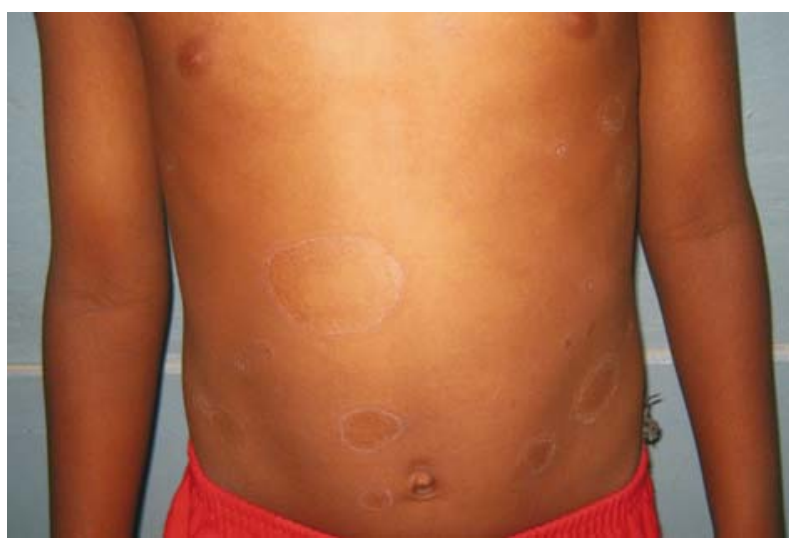

Fig.1 Mutiple annular erythema with trailing scale at inner border.

differential diagnosis includes those conditions that can have annular configuration and includes tinea, granuloma annulare, secondary syphilis, subacute cutaneous lupus erythematosus, erythema marginatum, erythema migrans, annular urticaria, and mycosis fungoides. The "trailing scale at inner border" of EAC is diagnostic. Most cases subside spontaneously.

AviJit Mondal, *Piyush Kumar and

\#RAJESH Kumar MaNDaL

Departments of Dermatology, College of Medicine and JNM Hospital, Kalyani, Nadia; *Katihar Medical College, Katihar, Bihar and ${ }^{\#}$ North Bengal Medical College. docpiyush@gmail.com 